\title{
An Early Warning Surveillance Platform for Developing Countries
}

\author{
Nsaibirni Robert Fondze $\mathrm{Jr}^{* 1,2}$, Gaëtan Texier, 3, Patrice Tchendjou², George Edouard \\ Kouamou$^{1}$, Richard Njouom ${ }^{2}$, Maurice Demanou ${ }^{2}$ and Maurice Tchuente ${ }^{1}$
}

${ }^{1}$ Department of Computer Science, Faculty of Science, University of Yaoundé I, Yaoundé, Cameroon; ${ }^{2}$ Centre Pasteur du Cameroun, Yaoundé, Cameroon; ${ }^{3}$ UMR 912 - SESSTIM - INSERM/IRD/Aix-Marseille Université, Marseille, France

\section{Objective}

Build a computer aided Early warning disease surveillance system adapted for Developing Countries (DCs) facing limited financial, human, intellectual, organizational, technological, and infrastructural resources.

\section{Introduction}

Early warning surveillance (EWS) is a key factor in the fight against tropical infectious diseases(1). However, the process of carrying out EWS is complex as it involves several actors and requires the use of diverse human, material and technological resources for data collection, analysis, and diffusion(2). Modern EWS systems make use of state of the art technologies and technics which require much financial input and adequate technological expertise for the users. More so, the culture and habits of users in DCs make it very difficult to run such EWS systems in this milieu. In this paper, we propose a generic early warning surveillance architecture that tackles the stages from just after data collection, through data analysis to feedback and that is adapted to the context of limited resource countries.

\section{Methods}

We proposed and designed an early warning disease surveillance architecture which makes use only of free and Open Source technologies and has five main components: The Data integration component that combines the multiple heterogeneous data sources into one physical repository (data warehouse) using Data Integration Tools (DIT), the Data Analysis component that comprises disease surveillance algorithms, the Task and Security management component, the Alarm-To-Alert component that associates the alarms produced with environmental, social, medical, and behavioral data using statistics, data mining and classification techniques to produce outbreak alerts as proposed in the Situational Diagnostics model by Chaudet et al.(3), and finally the Feedback component that produces real-time, intuitive and complete feedback for decision making purposes.

\section{Results}

We developed a prototype model of our architecture and started its implementation using the J2EE (Java Enterprise Edition) platform. To integrate the multiple heterogeneous data sources into a data warehouse, we choose to use Pentaho Data Integration, a free and open source DIT. Due to their statistics prowess, their ease of automation and their effectiveness in detecting outbreaks, we choose to write $\mathrm{R}$ scripts for the following four control chart based and/or regression model based algorithms: Farrington's algorithm(4), Serfling's algorithm(5), Exponentially Weighted Moving Averages (EWMA)(6), and Cumulative Sum (CUSUM) algorithms(1). This implementation is being carried out at the Centre Pasteur of Cameroon with cholera, yellow fever, and influenza as test diseases.

\section{Conclusions}

This platform is highly interoperable with a decentralized modular architecture that is prone to easily evolve due to its flexibility both at the data reception and data analysis stages. With a platform like this in place, there is a considerable gain in timeliness and accuracy in terms of data analysis and feedback. It is necessary however to invest in collecting appropriate data for early warning surveillance purposes.

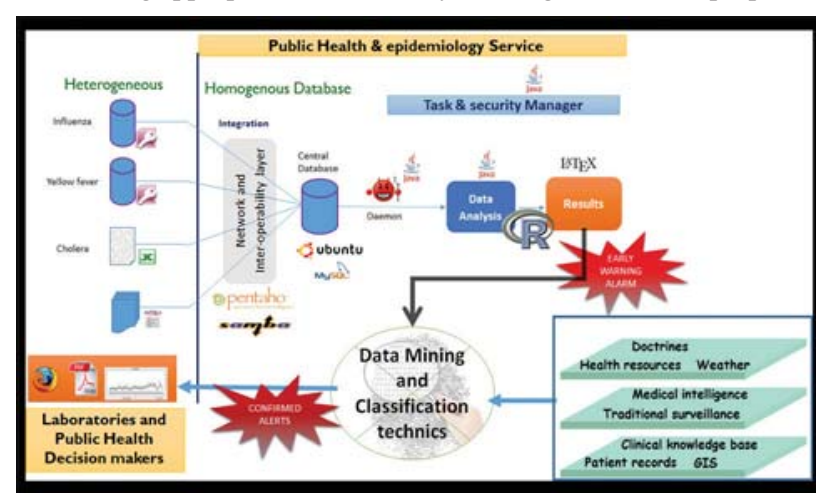

Keywords

Early warning disease surveillance; public health informatics; decision support

\section{Acknowledgments}

This work is supported by; CPC, the Department of Health and Human Services of the US, and the Institut Pasteur of Paris and is carried out at LIRIMA (Laboratoire International de Recherche en Informatique et en Mathématique Appliquée) University of Yaoundé I.

\section{References}

1. Joseph F. Lombardo DSB. Disease Surveillance: A Public Health Informatics Approach. John Wiley \& Sons, Inc, Hoboken, New Jersey;

2. Pascal Astagneau, Thierry Ancelle. Surveillance Epidemiologique. Medecine Sciences Publications; 2011.

3. Chaudet H, Pellegrin L, Gaudin C, Texier G, Queyriaux B, Meynard JB, Boutin J. A Model-Based Architecture for Supporting Situational Diagnosis in Real-Time Surveillance Systems. Adv Dis Surveill. 2007 152 ;

4. C.P. Farrington, N.J. Andrews, A. D. Beale, and M. A. Catchpole. A statistical algorithm for the early detection of outbreaks of infectious diseases. J R Stat Soc. 1996;547 - 563.

5. Robert E. Serfling. Methods for current statistical analysis of excess pneumoniainfluenza deaths. Public Health Reports,. 1963;

6. Lowry CA WW. A multivariate exponentially weighted moving average control chart. Technometrics; 1988.

\section{*Nsaibirni Robert Fondze Jr}

E-mail: nsairobby@gmail.com 